\title{
Optical properties of carbon nanotubes coated with orthogonal dipole switches
}

\author{
Antonio Setaro ${ }^{*, 1}$, Stefanie K. Kreft ${ }^{1}$, Michael Åxman Petersen ${ }^{2}$, Mogens Brøndsted Nielsen ${ }^{3}$, and \\ Stephanie Reich ${ }^{1}$
}

${ }^{1}$ Department of Physics of the Freie Universität Berlin, Arnimallee 14, 14195 Berlin, Germany

2 Institute for Chemistry, Technische Universität Berlin, Straße des 17. Juni 135, 10623 Berlin, Germany

${ }^{3}$ Institute for Chemistry, København University, Universitetsparken 5,2100 København, Danemark

* Corresponding author: e-mail setaro@physik.fu-berlin.de, Phone: +49 30838 56156, Fax: +49 3083856081

To disentangle the process of direct optical excitation of carbon nanotubes coated with photochromic switches from the isomerization of the latters, we functionalized single walled carbon nanotubes with the orthogonal dipole switching moiety Dihydroazulene / Vinylheptafulvene. The orthogonality of the switch decouples the kinetics of the dipole change from the optical excitation of the nanotubes, allowing the independent investigations of the excitation of tubes and switch.

1. Introduction Functionalizing carbon nanotubes with photocromic switching moieties offers the appealing advantage of creating a new class of hybrid nanosystems exhibiting the unique quantum features of carbon nanotubes (CNTs) and additional chance of affecting their properties by exposing them to light. Fast, reversible and highly efficient control over the modulation of the properties of the complexes can be achieved [1-4].

Over the last years, several attempts have been performed to yield such hybrids whilst preserving the nanotubes' features during the complexation process; covalent functionalization routines [1,3] have shown for example to achieve coverage of single- (SWNTs) and multi-walled carbon (MWNTs) for different purposes. The drawback of such approaches is the partial disruption of the carbon network due to the strongly perturbing covalent treatment, which results into the fading out of the tubes' quantum properties. No evidence of SWNTs' infrared emission from SWNTs, for example, could be provided, indicating

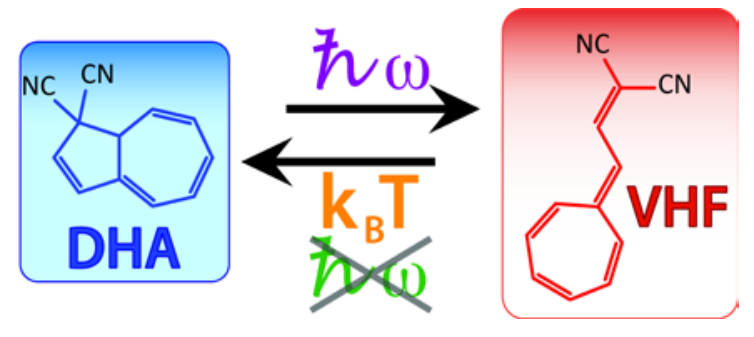

DHA to VHF conversion triggered by exposures to UV photons. The back isomerization is thermally activated and cannot be driven optically. strong suppression of the quantum properties of the nanocomplexes[3].

Recently, we showed a functionalization route for isolating and stabilizing SWNTs in solution while covering them with photo-switches [5]. The approach preserves the one-dimensional quantum nature of the nanotubes as proved by their bright emission. We also demonstrated that the switch-compound retained its switching ability when attached to the tube. Depending upon the physical property altered during switching process, several features of the nanotubes have been proven to be influenced in a controlled way. For example, by resorting to an azobenzene derivative, reversible isolation and stability of SWNTs in solution was demonstrated [6].

Switches that change their dipole moment offer the advantage of tuning the intensity and phenomenology of the Coulombic interaction between SWNTs and their adsorbate. This affects the optical and electronic properties of the nanotubes, red shifting their absorption bands, as predicted theoretically [7] and observed experimentally [8]. The pro- 
cess of rational design of the switching moiety is extremely important for such purposes, as the resulting morphology affects the switching performance. For example, overlap of the absorption bands of the switching moiety with other elements of the compound opens alternative de-excitation pathways. If fast enough, they might hinder the slower isomerization process. This was the case for an azobenzene derivative connected to a pyrene anchor [9]. Fast emission from pyrene made the azobenzene part unable to undergo any conformational change. By properly engineering the morphology of a switching moiety based on spiropyran/merocyanine, we affected the strength of the interaction between SWNTs and dipoles [10]. When tailoring the morphology of the anchor part of the compound, on the other hand, we manipulated the chirality distributions of the SWNTs in the complex [11-13]. The drawback of spiropyran is that the isomerization in both directions is triggered by exposure to radiation (UV for the spiro-to-mero conversion and visible for the mero-to-spiro back conversion). The excitation windows of SWNTs lies in the spectral region where mero-to-spiro back isomerization occurs. This hinders investigation of the emission features of the nanotubes in the high dipole moment form (merocyanine) of the switch.

Here we propose to use a different kind of dipole switch, namely the dihydroazulene/vinylheptafulvene (DHA/VHF) system. It is an orthogonal switch due to the occurrence of a conical intersection in its energy landscape $[14,15]$. The DHA-to-VHF isomerization is triggered by the exposure to UV photons while the VHF-to-DHA backisomerization is thermally driven and is not perturbed by the exposure of photons in the visible range. This allows investigation of the emission properties of SWNTs in both DHA as well as VHF state. Another advantage of DHA/VHF relies in the big difference of the dipole moment between the two states: In the DHA form the compound is neutral, exhibiting no appreciable dipole moment, while in the VHF form gets a dipole moment up to $20 \mathrm{D}$.

2. Experiment and discussion Dihydroazulene is an organic compound first synthesized by Daub et al. [16]. It undergoes a 10-electron retroelectrocyclization under UV irradiation and converts into the ring-opened 8-VinylHeptafulvene. The back reaction is thermally driven. The switching process is accompanied by a change of the absorption of the molecule with consequent color change from yellow to dark red. The DHA cromophore has an alternating, conjugated $\pi$-system. The irradiation induces a ring-opening of the five-ring and a cis-trans isomerization, leading to an extended conjugated but nonalternant $\pi$ system. The ring-opening is accompanied by additional structural changes. The butadien in VHF is in the s-trans confirmation and is twisted about the C-C bond. [17]. A sketch of the molecule synthesized for the present work can be found in Fig. 1. It comprises a head and a tail. The

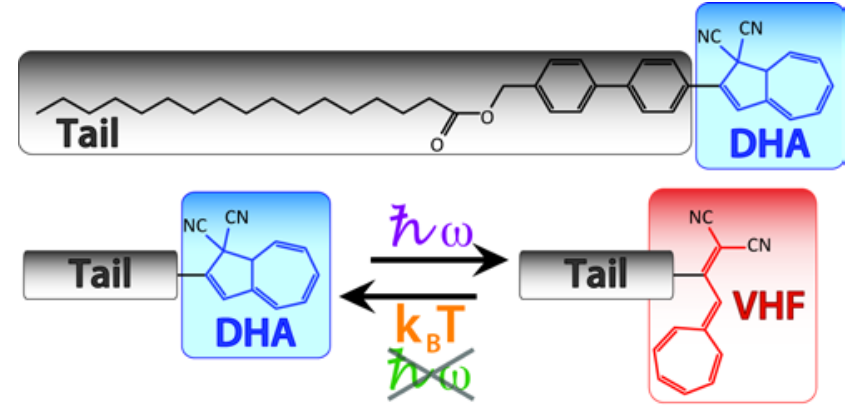

Figure 1 Schematic depiction of our switching moiety.

tail is composed of a carbon chain $\left(\mathrm{C}_{16}\right)$ and a biphenyl moiety. It provides the SWNTs isolating and solubilizing ability to the compound. The head part contains the dipole switch that passes from the DHA to the VHF form under UV irradiation $(\lambda=367 \mathrm{~nm})$. Details on the synthesis of our compound will be object of future publication. The length of the chain was chosen to separate the dipole switch from the tubes and avoid spurious effects due, for example, to $\pi$ $\pi$ interactions between the head and the tubes that could hinder the isomerization [10]. The expected head-tubes separation is $3 \mathrm{~nm}$. The synthesis of the tube-switch complexes follows the functionalization of SWNTs with spiroderivatives in THF. It requires several sonication and centrifugation steps; for details refer to Ref. [5]. The starting molarity of the switching compound in our suspensions was $5 \mu \mathrm{M}$.

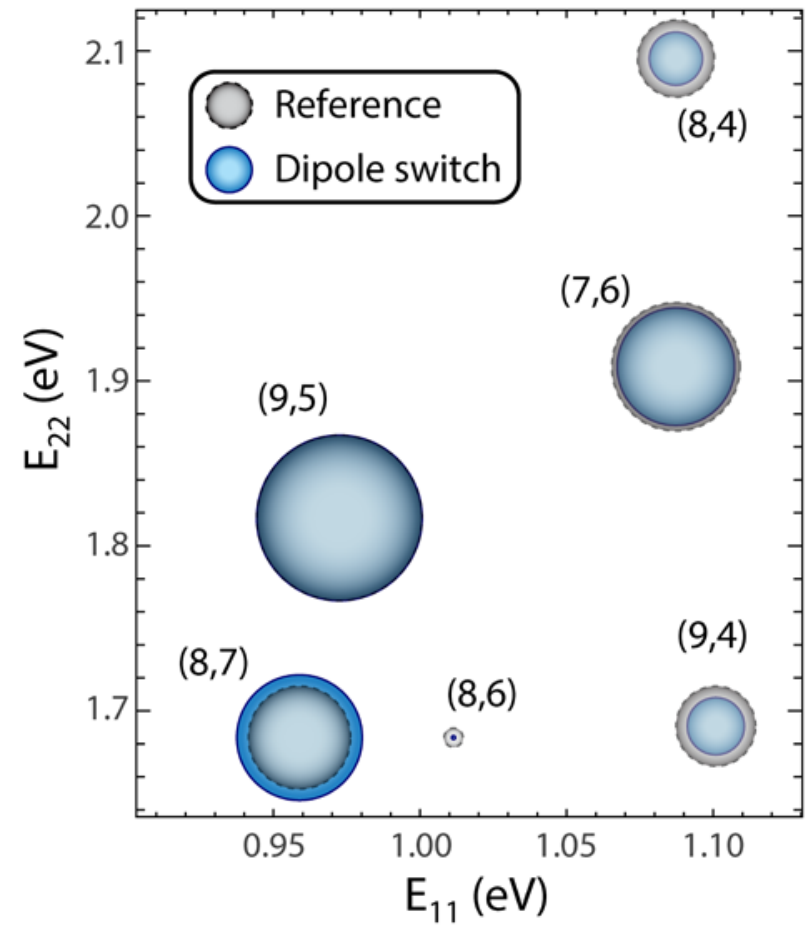

Figure 2 Pseudo-PLE map of SWNTs functionalized with the reference compound (gray circles, dashed contour) and with the switching moiety (blue circles, continuous contour). 
(a)

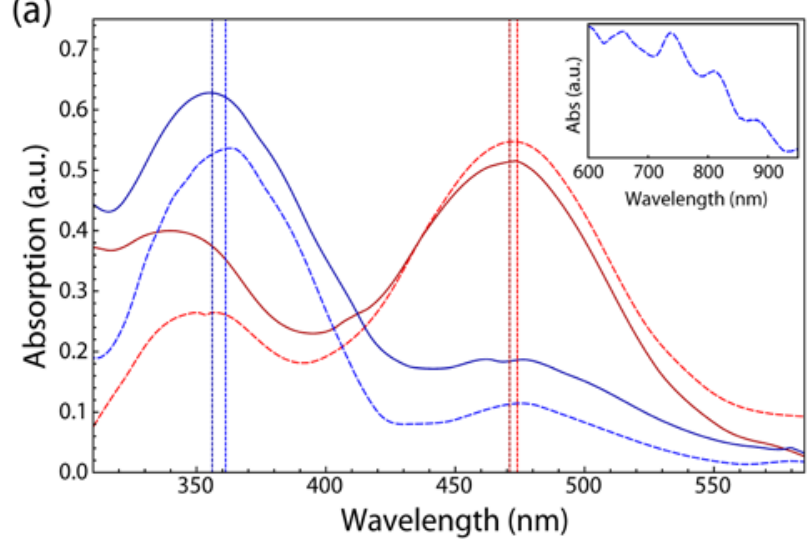

(b)

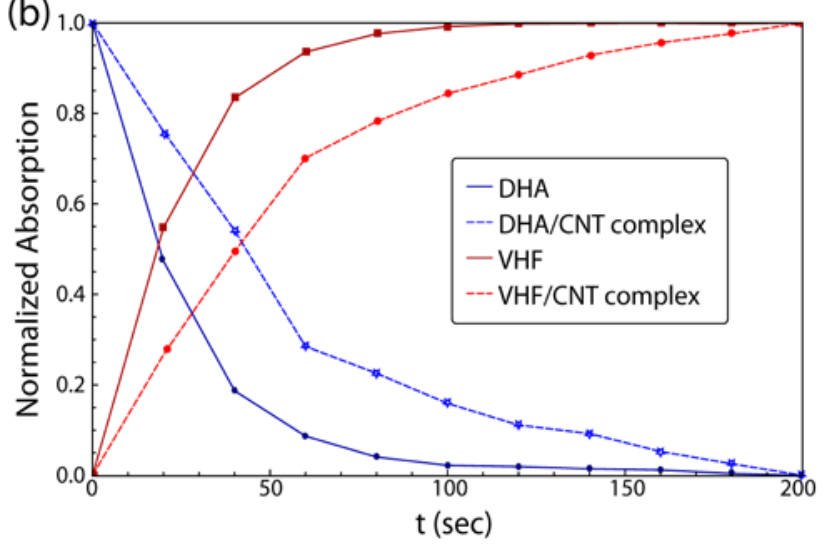

Figure 3 (a) Absorption spectra of the switching moiety in the DHA (blue) and VHF state state of the pure molecules (continuos lines) and of the hybrid molecule-nanotube systems (dashed lines). In the inset the absorption of the semiconducting tubes in the $\mathrm{S}_{22}$ region is reported. (b) Intensity of the absorption bands during the isomerization process.

To isolate the effect of the dipole switch in our compounds we also functionalized nanotubes only with the tail part $\left(\mathrm{C}_{16}\right.$ chain and biphenyl moiety) without DHA. Both compounds isolated and solubilized SWNTs, as proven by the bright emission from our final suspensions. The dipole head in its neutral DHA form does not affect the spectral positions of the investigated tubes. This can be seen in the pseudo-PLE map in Fig. 2 where we compare the emission energies of tubes functionalized with the reference compound and with our switching moiety. The radii of the spots are proportional to the intensity of the emitted radiation; the intensities of the emission from the two samples were normalized to the emission of the brightest tube (the $(9,5)$ chirality) for allowing direct comparison. Unfortunately, the chain results in such big separation between tube and dipole switch that no significant change in the PLE peaks was observed when inducing the dipole moment by passing to the VHF form of the compound. This is consistent with analogous response observed in the spiropyran-nanotubes complexes, where the interaction between tube and dipole started strengthening for separations below $1 \mathrm{~nm}[3,5]$. Despite the change of the spectral position of the tubes being too small to be observed, the complex formation does affect the switch. In Fig. 3(a) it can be seen how the DHA absorption band (360 nm) is red-shifted by $67 \mathrm{meV}$ as a consequence of the interaction with the SWNTs. Analogously, the VHF maximum shifts by 34 $\mathrm{meV}$. A similar behaviour has been observed for the spiropyran/merocyanine system [10].

The most striking effect of the DHA-nanotube interaction is observed in the switching ability of the compounds. In Fig. 3(b) we show the temporal response of the intensity of the DHA and VHF bands pure and after complexation with the SWNTs. Table 1 reports the time constants of the isomerization process, that are strongly altered by the interaction with the tubes.
This effect has no equivalent in the analogous spiropyran/merocyanine configuration, where for big tube-dipole separation $(\mathrm{d}>1 \mathrm{~nm})$ the isomerization dynamics is not affected by the presence of the tubes [3,5]. This slower isomerization kinetics occurs only when forming the tubeswitch hybrid; to rule out that the enhanced absorption due to the presence of the nanotubes causes this slowing down, we performed a control experiment placing an additional cuvette filled with a reference nanotube sample in front of the UV source. The reference sample contains no switching moiety and the amount of tubes in suspension was adjusted to achieve the same optical density of the suspension containing the dipole-tubes complexes. Table 1 reports the isomerization times of the pure compound exposed to the UV source (labelled as "pure"), of the pure compound with the reference sample placed between UV source and sample (labelled as "blocked") and of the tube-switch hybrids suspension (labelled as "complex"). The presence of the reference sample does not significantly alter the isomerization rate of the free compound. We can thus conclude from the control experiment that the slowing down of the isomerization rate is due to the tube-switch interaction within the complexes.

The effect on the transition-state of the VHF by the presence of SWNTs is in line with previous observations $[18,19]$, where it was shown that the electronic nature of substituents on DHA/VHF system greatly affects the rate of the thermal back reaction.

Table 1 Isomerization times of our compound in different configurations.

\begin{tabular}{ccc}
\hline & $\boldsymbol{\tau}_{\text {DHA }}(\mathbf{s})$ & $\boldsymbol{\tau}_{\text {VHF }}(\mathbf{s})$ \\
\hline pure & $52 \pm 1$ & $46 \pm 1$ \\
blocked & $59 \pm 1$ & $55 \pm 2$ \\
complex & $77 \pm 3$ & $75 \pm 3$ \\
\hline
\end{tabular}


3. Conclusions SWNTs were functionalized with orthogonal dipole switches taking advantage of the exclusively thermally back-isomerization process. No interfering effects arise from exposure to visible radiation, ensuring the chance of performing fundamental optical investigations of the SWNTs properties without perturbing the state of the switch. The formation of the switch-nanotube hybrid structures affects the isomerization kinetics of the molecular switch. Appreciable effects on the optical properties of the nanotubes would benefit of further optimization of the morphology of the compound, addressing in particular the length of the tail part of the compound for reducing the tube-dipole separation.

Acknowledgements This work has been supported by the DFG under the SfB 658. A.S. gratefully thanks the FU Focus Area NanoScale for financial support. M.A.P. thanks the Danish Research Council for Independent Research|Natural Sciences, The Carlsberg Foundation.

\section{References}

[1] R.F. Khairutdinov, M.E. Itkis, and R.C. Haddon, Nano Lett. 4, 1529 (2004).

[2] X. Guo, L. Huang, S. O’Brien, P. Kim, and C. Nuckolls, JACS 127, 15045 (2005).

[3] E. Del Canto, K. Flavin, M. Natali, T. Perova, and S. Giordani, Carbon 48, 2815 (2010).

[4] X. Zhou, T. Zifer, B.M Wong, K.L. Krafcik, F. Léonard, and A.L. Vance, Nano Lett. 9, 1028 (2009).

[5] A. Setaro, P. Bluemmel, C Maity, S Hecht, and S. Reich, Adv. Funct. Mat. 22, 2425 (2012).
[6] C. Kördel, A. Setaro, P. Bluemmel, C.S. Popeney, S. Reich, and R. Haag, Nanoscale 4, 3029 (2012).

[7] E. Malic, C. Weber, M. Richter, V. Atalla, T. Klamroth, P. Saalfrank, S. Reich, and A. Knorr, PRL 106, 097401 (2011).

[8] E Malic, A Setaro, P Bluemmel, C. F Sanz-Navarro, P. Ordejon, S Reich, and A Knorr, Journ. Phys: Cond Matt. 24, 394006 (2012).

[9] P. Bluemmel, A. Setaro, C.S. Popeney, R. Haag, and S. Reich, Phys. Stat. Sol. B 247, 2891 (2010).

[10]P. Bluemmel, A. Setaro, C. Maity, S. Hecht, and S. Reich Journ. Phys: Cond Matt. 24, 394005 (2012).

[11]P. Bluemmel, A. Setaro, C. Maity, S. Hecht, and S. Reich Phys. Stat. Sol. B 249, 2479 (2012).

[12]A. Setaro, C. S. Popeney, B. Trappmann, V. Datsyuk, R. Haag, and S. Reich, Chem. Phys. Lett. 493, 147 (2010).

[13] C. S. Popeney, A. Setaro, R. C. Mutihac, P. Bluemmel, B. Trappmann, J. Vonneman, S. Reich, and R. Haag, ChemPhysChem 13, 203 (2012).

[14] J. Ern, M. Petermann, T. Mrozek, J. Daub, and K. Kuldov, Chem. Phys. 259, 331 (2000).

[15] M. Boggio-Pasqua, M. J. Bearpark, P. A. Hunt, and M. A. Robb, JACS 124, 1456 (2002).

[16] J. Daub T. Knöchel, and A. Mannschreck, Ang. Chem. Int. Ed. Engl. 23, 12 (1984).

[17] J. Daub, S. Gierisch, U. Klement, T. Knochel, G. Maasb, and U. Seitz, Chem. Ber. 119, 2631 (1986).

[18] S.L. Broman, M.Å. Petersen, C.G. Tortzen, A. Kadziola, K. Kilså, and M.B. Nielsen, J. Am. Chem. Soc., 132, 9165 (2010).

[19] M. Å Petersen, S.L. Broman, K. Kilså, A. Kadziola, and M.B. Nielsen, Eur. J. Org. Chem., 6, 1033 (2011). 